\title{
NON-ASSOCIATIVE ORE EXTENSIONS
}

\author{
PATRIK NYSTEDT \\ University West, Department of Engineering Science, SE-46186 Trollhättan, Sweden
}

JOHAN ÖINERT

Blekinge Institute of Technology, Department of Mathematics and Natural Sciences, SE-37179 Karlskrona, Sweden

JOHAN RICHTER

Mälardalen University, Academy of Education, Culture and Communication, Box 883, SE-72123 Västerås, Sweden

\begin{abstract}
We introduce non-associative Ore extensions, $S=R[X ; \sigma, \delta]$, for any nonassociative unital ring $R$ and any additive maps $\sigma, \delta: R \rightarrow R$ satisfying $\sigma(1)=1$ and $\delta(1)=0$. In the special case when $\delta$ is either left or right $R_{\delta}$-linear, where $R_{\delta}=\operatorname{ker}(\delta)$, and $R$ is $\delta$-simple, i.e. $\{0\}$ and $R$ are the only $\delta$-invariant ideals of $R$, we determine the ideal structure of the non-associative differential polynomial ring $D=R\left[X ; \mathrm{id}_{R}, \delta\right]$. Namely, in that case, we show that all ideals of $D$ are generated by monic polynomials in the center $Z(D)$ of $D$. We also show that $Z(D)=R_{\delta}[p]$ for a monic $p \in R_{\delta}[X]$, unique up to addition of elements from $Z(R)_{\delta}$. Thereby, we generalize classical results by Amitsur on differential polynomial rings defined by derivations on associative and simple rings. Furthermore, we use the ideal structure of $D$ to show that $D$ is simple if and only if $R$ is $\delta$-simple and $Z(D)$ equals the field $R_{\delta} \cap Z(R)$. This provides us with a non-associative generalization of a result by Öinert, Richter, and Silvestrov. This result is in turn used to show a non-associative version of a classical result by Jordan concerning simplicity of $D$ in the cases when the characteristic of the field $R_{\delta} \cap Z(R)$ is either zero or a prime. We use our findings to show simplicity results for both non-associative versions of Weyl algebras and non-associative differential polynomial rings defined by monoid/group actions on compact Hausdorff spaces.
\end{abstract}

\section{INTRODUCTION}

In 1933 Ore 23] introduced a version of non-commutative polynomial rings, nowadays called Ore extensions, that have become one of the most useful constructions in ring theory. The Ore extensions play an important role when investigating cyclic algebras, enveloping rings of solvable Lie algebras, and various types of graded rings such as group rings and crossed

E-mail addresses: patrik.nystedt@hv.se; johan.oinert@bth.se; johan.richter@mdh.se. 2010 Mathematics Subject Classification. 17D99, 17A36, 17A99, 16S36, 16W70, 16 U70.

Key words and phrases. non-associative Ore extension, simple, outer derivation. 
products, see e.g. [7, 14, [18 and [27]. They are also a natural source of examples and counter-examples in ring theory, see e.g. 44 and 6]. Furthermore, various special cases of Ore extensions are used as tools in diverse analytical settings, such as differential-, pseudodifferential and fractional differential operator rings [10] and $q$-Heisenberg algebras [12].

Let us recall the definition of an (associative) Ore extension. Let $S$ be a unital ring. Take $x \in S$ and let $R$ be a subring of $S$ containing 1, the multiplicative identity element of $S$.

Definition 1. The pair $(S, x)$ is called an Ore extension of $R$ if the following axioms hold:

(O1) $S$ is a free left $R$-module with basis $\left\{1, x, x^{2}, \ldots\right\}$;

(O2) $x R \subseteq R+R x$;

(O3) $S$ is associative.

If $(\mathrm{O} 2)$ is replaced by

$(\mathrm{O} 2)^{\prime}[x, R] \subseteq R$;

then $(S, x)$ is called a differential polynomial ring over $R$.

Recall that $[x, R]$ denotes the set of finite sums of elements of the form $[x, r]=x r-r x$, for $r \in R$.

To construct Ore extensions, one considers generalized polynomial rings $R[X ; \sigma, \delta]$ over an associative ring $R$, where $\sigma$ is a ring endomorphism of $R$, respecting 1 , and $\delta$ is a $\sigma$-derivation of $R$, i.e. an additive map $R \rightarrow R$ satisfying $\delta(a b)=\sigma(a) \delta(b)+\delta(a) b$, for $a, b \in R$. Let $\mathbb{N}$ denote the set of non-negative integers. As an additive group $R[X ; \sigma, \delta]$ is equal to the usual polynomial ring $R[X]$. The ring structure on $R[X ; \sigma, \delta]$ is defined on monomials by

$$
a X^{m} \cdot b X^{n}=\sum_{i \in \mathbb{N}} a \pi_{i}^{m}(b) X^{i+n},
$$

for $a, b \in R$ and $m, n \in \mathbb{N}$, where $\pi_{i}^{m}$ denotes the sum of all the $\left(\begin{array}{c}m \\ i\end{array}\right)$ possible compositions of $i$ copies of $\sigma$ and $m-i$ copies of $\delta$ in arbitrary order (see equation (11) in [23]). Here we make the convention that $\pi_{i}^{m}(b)=0$, for $i, m \in \mathbb{N}$ such that $i>m$. The product (1) makes the pair $(R[X ; \sigma, \delta], X)$ an Ore extension of $R$. In fact, (O1) and (O2) are immediate and (O3) can be shown in several different ways, see e.g. [5, Proposition 7.1], [19], [26], [27, Proposition 1.6.15], or Proposition 17 in the present article for yet another proof. This class of generalized polynomial rings provides us with all Ore extensions of $R$. Indeed, given an Ore extension $(S, x)$ of $R$, then define the maps $\sigma: R \rightarrow R$ and $\delta: R \rightarrow R$ by the relations $x a=\delta(a)+\sigma(a) x$, for $a \in R$. Then it follows that $\sigma$ is a ring endomorphism of $R$, respecting $1, \delta$ is a $\sigma$-derivation of $R$ and there is a unique well defined ring isomorphism $f: S \rightarrow R[X ; \sigma, \delta]$ subject to the relations $f(x)=X$ and $\left.f\right|_{R}=\operatorname{id}_{R}$. If $(S, x)$ is a differential polynomial ring over $R$, then $\sigma=\operatorname{id}_{R}$ and $\delta$ is a derivation on $R$.

Many different properties of associative Ore extensions, such as when they are integral domains, principal domains, prime or noetherian have been studied by numerous authors (see e.g. [8] or [18 for surveys). Here we focus on the property of simplicity of differential polynomial rings $D=R\left[X ; \operatorname{id}_{R}, \delta\right]$. Recall that $\delta$ is called inner if there is $a \in R$ such that $\delta(r)=a r-r a$, for $r \in R$. In that case we write $\delta=\delta_{a}$. If $\delta$ is not inner, then $\delta$ is called outer. We let the characteristic of a ring $R$ be denoted by $\operatorname{char}(R)$. In an early article by Jacobson [13] it is shown that if $\delta$ is outer and $R$ is a division $\operatorname{ring}$ with $\operatorname{char}(R)=0$, then $D$ is simple. The case of positive characteristic is more complicated and $D$ may contain non-trivial ideals. In fact, Amitsur [1] has shown that if $R$ is a division $\operatorname{ring}$ with $\operatorname{char}(R)=p>0$, then every ideal of $D$ is generated by a polynomial, all of whose monomials have degrees which are 
multiples of $p$. A few years later Amitsur 2] generalized this result to the case of simple $R$. To describe this generalization we need to introduce some more notation. Let $T$ be a subring of $D$. Let $Z(T)$ denote the center of $T$, i.e. the set of all elements in $T$ that commute with every element of $T$. If $T$ is a subring of $R$, then put $T_{\delta}=T \cap \operatorname{ker}(\delta)$. Note that if $R$ is simple, then $Z(R)$ is a field. Therefore, in that case, $\operatorname{char}(R)=\operatorname{char}(Z(R))$ and hence $\operatorname{char}(R)$ is either zero or a prime $p>0$.

Theorem 2 (Amitsur [2]). Suppose that $R$ is a simple associative ring and let $\delta$ be a derivation on $R$. If we put $D=R\left[X ; \mathrm{id}_{R}, \delta\right]$, then the following assertions hold:

(a) Every ideal of $D$ is generated by a unique monic polynomial in $Z(D)$;

(b) There is a monic $b \in R_{\delta}[X]$, unique up to addition of elements from $Z(R)_{\delta}$, such that $Z(D)=Z(R)_{\delta}[b]$;

(c) If $\operatorname{char}(R)=0$ and $b \neq 1$, then there is $c \in R_{\delta}$ such that $b=c+X$. In that case, $\delta=\delta_{c}$

(d) If $\operatorname{char}(R)=p>0$ and $b \neq 1$, then there is $c \in R_{\delta}$ and $b_{0}, \ldots, b_{n} \in Z(R)_{\delta}$, with $b_{n}=1$, such that $b=c+\sum_{i=0}^{n} b_{i} X^{p^{i}}$. In that case, $\sum_{i=0}^{n} b_{i} \delta^{p^{i}}=\delta_{c}$.

The condition that $R$ is simple in the above theorem is not necessary for simplicity of $D=R\left[X ; \mathrm{id}_{R}, \delta\right]$. Consider e.g. the well known example of the first Weyl algebra where $R=K[Y], K$ is a field with $\operatorname{char}(K)=0$ and $\delta$ is the usual derivative on $R$ (for more details, see e.g. [27, Example 1.6.32]). However, $\delta$-simplicity of $R$ is always a necessary condition for simplicity of $D$ (see [15, Lemma 4.1.3(i)] or Proposition 21). Recall that an ideal $I$ of $R$ is called $\delta$-invariant if $\delta(I) \subseteq I$. The ring $R$ is called $\delta$-simple if $\{0\}$ and $R$ are the only $\delta$-invariant ideals of $R$. Note that if $R$ is $\delta$-simple, then the $\operatorname{ring} Z(R)_{\delta}$ is always a field. Therefore, in that case, $\operatorname{char}(R)=\operatorname{char}\left(Z(R)_{\delta}\right)$ and hence $\operatorname{char}(R)$ is either zero or a prime $p>0$. Jordan [15] (and Cozzens and Faith [8] in a special case) has shown the following result.

Theorem 3 (Jordan [15]). Suppose that $R$ is a $\delta$-simple associative ring and let $\delta$ be a derivation on $R$. If we put $D=R\left[X ; \mathrm{id}_{R}, \delta\right]$, then the following assertions hold:

(a) If $\operatorname{char}(R)=0$, then $D$ is simple if and only if $\delta$ is outer;

(b) If $\operatorname{char}(R)=p>0$, then $D$ is simple if and only if no derivation of the form $\sum_{i=0}^{n} b_{i} \delta^{p^{i}}$, $b_{i} \in Z(R)_{\delta}$, and $b_{n}=1$, is an inner derivation induced by an element in $R_{\delta}$.

In the case when $R$ is commutative, Cozzens and Faith [8] (for integral domains $R$ of prime characteristic) and Goodearl and Warfield [11] (in the general case) have shown that $R\left[x ; \mathrm{id}_{R}, \delta\right]$ is simple if and only if $R$ is $\delta$-simple and $R$ is infinite-dimensional as a vector space over $R_{\delta}$. If one has a family of commuting derivations, then one can form a differential polynomial ring in several variables. The articles [17], 24] and [28] consider the question when such rings are simple. In the preprint [20] the authors of the present article study when non-associative differential polynomial rings in several variables are simple.

In the simplicity results mentioned above, a distinction is often made between the cases when the characteristic of $R$ is zero or the characteristic of $R$ is prime. Special attention is also often paid to the case when $R$ is commutative. However, in 22 Öinert, Richter and Silvestrov have shown the following simplicity result that holds for all associative differential polynomial rings regardless of characteristic.

Theorem 4 (Öinert, Richter and Silvestrov [22]). If $R$ is associative and $\delta: R \rightarrow R$ is a derivation, then $D=R\left[X ; \operatorname{id}_{R}, \delta\right]$ is simple if and only if $R$ is $\delta$-simple and $Z(D)$ is a field. 
In this article, we address the question of what it should mean for a pair $(S, x)$ to be a nonassociative Ore extension of $R$ and when the resulting rings are simple. It seems to the authors of the present article that this question has not previously been analysed in the literature. Let us briefly describe the train of reasoning that lead the authors to their definition of such objects. The product (10) equips the set $R[X ; \sigma, \delta]$ of generalized polynomials over any nonassociative ring $R$ with a well defined non-associative ring structure for any additive maps $\sigma: R \rightarrow R$ and $\delta: R \rightarrow R$ satisfying $\sigma(1)=1$ and $\delta(1)=0$. We wish to adapt the axioms (O1), (O2) and (O3) to the non-associative situation so that the resulting collection of nonassociative rings coincides with this family of generalized polynomial rings. It turns out that this happens precisely when $x$ belongs to the right and middle nucleus of $S$. To be more precise, let $S$ be a non-associative ring, by this we mean that $S$ is an additive abelian group equipped with a multiplication which is distributive with respect to addition and which has multiplicative identity 1 . We suggest the following.

Definition 5. The pair $(S, x)$ is called a non-associative Ore extension of $R$ if the following axioms hold:

(N1) $S$ is a free left $R$-module with basis $\left\{1, x, x^{2}, \ldots\right\}$;

(N2) $x R \subseteq R+R x$;

(N3) $(S, S, x)=(S, x, S)=\{0\}$.

If (N2) is replaced by

$(\mathrm{N} 2)^{\prime}[x, R] \subseteq R$;

then $(S, x)$ is called a non-associative differential polynomial ring over $R$.

For non-empty subsets $A, B$ and $C$ of $S$, we let $(A, B, C)$ denote the set of finite sums of elements of the form $(a, b, c)=(a b) c-a(b c)$, for $a \in A, b \in B$ and $c \in C$. Note that from (N3) it follows that the element $x$ is power associative, so that the symbols $x^{i}$, for $i \in \mathbb{N}$, are well defined.

Here is an outline of this article.

In Section 2, we gather some well known facts from non-associative ring and module theory that we need in the sequel. In particular, we state our conventions concerning modules over non-associative rings and what a basis should mean in that situation.

In Section 3, we show that there is a bijection between the set of non-associative Ore extensions of $R$ and the set of generalized polynomial rings $R[X ; \sigma, \delta]$ over $R$, where $\sigma$ and $\delta$ are additive maps $R \rightarrow R$ such that $\sigma(1)=1$ and $\delta(1)=0$. If $T$ is a subset of $R$, then we put $T_{\delta}^{\sigma}=\{a \in T \mid \sigma(a)=a$ and $\delta(a)=0\}, T_{\delta}=T_{\delta}^{\mathrm{id}_{R}}$ and $T^{\sigma}=T_{0}^{\sigma}$. In Section 3 , we introduce the class of strong non-associative Ore extensions (see Definition 12). These correspond to generalized polynomial rings $R[X ; \sigma, \delta]$, where $\sigma$ is a, what we call, fixed point homomorphism of $R$ and $\delta$ is a, what we call, $\sigma$-kernel derivation of $R$. By this we mean that $\sigma$ and $\delta$ are maps $R \rightarrow R$ satisfying $\sigma(1)=1, \delta(1)=0$ and both of them are right $R_{\delta}^{\sigma}$-linear or both of them are left $R_{\delta}^{\sigma}$-linear. Clearly, every classical derivation is a $\sigma$-kernel derivation with $\sigma=\mathrm{id}_{R}$ and every classical homomorphism is a fixed point homomorphism. In general, a $\sigma$-kernel derivation with $\sigma=\mathrm{id}_{R}$ will simply be called a kernel derivation.

In Section 4, we introduce $\sigma$ - $\delta$-simplicity for rings $R$, where $\sigma$ and $\delta$ are additive maps $R \rightarrow R$ such that $\sigma(1)=1$ and $\delta(1)=0$ (see Definition 20). We show that $\sigma$ - $\delta$-simplicity of $R$ is a necessary condition for simplicity of non-associative Ore extensions $R[X ; \sigma, \delta]$ (see Proposition 21). We also show that if $R$ is $\sigma$ - $\delta$-simple, then $Z(R)_{\delta}^{\sigma}$ is a field (see Proposition 221). Thus, in that case, we get that $\operatorname{char}(R)=\operatorname{char}\left(Z(R)_{\delta}^{\sigma}\right)$ and hence that $\operatorname{char}(R)$ is either 
zero or a prime $p>0$. In Section 4 , we prove the following non-associative generalization of Theorems 2, 3 and 4 .

Theorem 6. Suppose that $R$ is a non-associative ring and that $\delta$ is a kernel derivation on $R$. If we put $D=R\left[X ; \mathrm{id}_{R}, \delta\right]$, then the following assertions hold:

(a) If $R$ is $\delta$-simple, then every ideal of $D$ is generated by a unique monic polynomial in $Z(D)$;

(b) If $R$ is $\delta$-simple, then there is a monic $b \in R_{\delta}[X]$, unique up to addition of elements from $Z(R)_{\delta}$, such that $Z(D)=Z(R)_{\delta}[b]$;

(c) $D$ is simple if and only if $R$ is $\delta$-simple and $Z(D)$ is a field. In that case $Z(D)=Z(R)_{\delta}$ in which case $b=1$;

(d) If $R$ is $\delta$-simple, $\delta$ is a derivation on $R$ and $\operatorname{char}(R)=0$, then either $b=1$ or there is $c \in R_{\delta}$ such that $b=c+X$. In the latter case, $\delta=\delta_{c}$;

(e) If $R$ is $\delta$-simple, $\delta$ is a derivation on $R$ and $\operatorname{char}(R)=p>0$, then either $b=1$ or there is $c \in R_{\delta}$ and $b_{0}, \ldots, b_{n} \in Z(R)_{\delta}$, with $b_{n}=1$, such that $b=c+\sum_{i=0}^{n} b_{i} X^{p^{i}}$. In the latter case, $\sum_{i=0}^{n} b_{i} \delta^{p^{i}}=\delta_{c}$.

In Section 5, we introduce non-associative versions of the first Weyl algebra (see Definition 301) and we show that they are often simple regardless of the characteristic (see Theorem 32).

In Section 6, we introduce a special class of $\sigma$-kernel derivations induced by ring automorphisms (see Definition 34). This yields simplicity results for a differential polynomial ring analogue of the quantum plane (see Theorem 37) and for differential polynomial rings defined by monoid/group actions on compact Hausdorff spaces (see Theorem 40 and Theorem 42).

In Section 77 we show that if the coefficients are associative, then we can often obtain simplicity of the differential polynomial ring just from the assumption that the map $\delta$ is not a derivation.

\section{Preliminaries from Non-Associative Ring Theory}

In this section, we recall some notions from non-associative ring theory that we need in subsequent sections. Although the results stated in this section are presumably rather well known, we have, for the convenience of the reader, nevertheless chosen to include proofs of these statements.

Throughout this section, $R$ denotes a non-associative ring. By this we mean that $R$ is an additive abelian group in which a multiplication is defined, satisfying left and right distributivity. We always assume that $R$ is unital and that the multiplicative identity of $R$ is denoted by 1. The term "non-associative" should be interpreted as "not necessarily associative". Therefore all associative rings are non-associative. If a ring is not associative, we will use the term "not associative ring".

By a left module over $R$ we mean an additive group $M$ equipped with a biadditive map $R \times M \ni(r, m) \mapsto r m \in M$. In that case, we say that a subset $B$ of $M$ is a basis if for every $m \in M$, there are unique $r_{b} \in R$, for $b \in B$, such that $r_{b}=0$ for all but finitely many $b \in B$, and $m=\sum_{b \in B} r_{b} b$. Right modules over $R$ and bases are defined in an analogous manner.

Recall that the commutator $[\cdot, \cdot]: R \times R \rightarrow R$ and the associator $(\cdot, \cdot, \cdot): R \times R \times R \rightarrow R$ are defined by $[r, s]=r s-s r$ and $(r, s, t)=(r s) t-r(s t)$ for all $r, s, t \in R$, respectively. The commuter of $R$, denoted by $C(R)$, is the subset of $R$ consisting of elements $r \in R$ such that $[r, s]=0$ for all $s \in R$. The left, middle and right nucleus of $R$, denoted by $N_{l}(R)$, $N_{m}(R)$ and $N_{r}(R)$, respectively, are defined by $N_{l}(R)=\{r \in R \mid(r, s, t)=0$, for $s, t \in R\}$, 
$N_{m}(R)=\{s \in R \mid(r, s, t)=0$, for $r, t \in R\}$, and $N_{r}(R)=\{t \in R \mid(r, s, t)=0$, for $r, s \in R\}$. The nucleus of $R$, denoted by $N(R)$, is defined to be equal to $N_{l}(R) \cap N_{m}(R) \cap N_{r}(R)$. From the so-called associator identity $u(r, s, t)+(u, r, s) t+(u, r s, t)=(u r, s, t)+(u, r, s t)$, which holds for all $u, r, s, t \in R$, it follows that all of the subsets $N_{l}(R), N_{m}(R), N_{r}(R)$ and $N(R)$ are associative subrings of $R$. The center of $R$, denoted by $Z(R)$, is defined to be equal to the intersection $N(R) \cap C(R)$. It follows immediately that $Z(R)$ is an associative, unital and commutative subring of $R$.

Proposition 7. The following three equalities hold:

$$
\begin{aligned}
& Z(R)=C(R) \cap N_{l}(R) \cap N_{m}(R) ; \\
& Z(R)=C(R) \cap N_{l}(R) \cap N_{r}(R) ; \\
& Z(R)=C(R) \cap N_{m}(R) \cap N_{r}(R) .
\end{aligned}
$$

Proof. We only show (2). The equalities (3) and (4) are shown in a similar way and are therefore left to the reader. It is clear that $Z(R) \subseteq C(R) \cap N_{l}(R) \cap N_{m}(R)$. Now we show the reversed inclusion. Take $r \in C(R) \cap N_{l}(R) \cap N_{m}(R)$. We need to show that $r \in N_{r}(R)$. Take $s, t \in R$. We wish to show that $(s, t, r)=0$, i.e. $(s t) r=s(t r)$. Using that $r \in$ $C(R) \cap N_{l}(R) \cap N_{m}(R)$ we get $(s t) r=r(s t)=(r s) t=(s r) t=s(r t)=s(t r)$.

Proposition 8. If $r \in Z(R)$ and $s \in R$ satisfy $r s=1$, then $s \in Z(R)$.

Proof. Let $r \in Z(R)$ and suppose that $r s=1$. First we show that $s \in C(R)$. To this end, take $u \in R$. Then $s u=(s u) 1=(s u)(r s)=(r(s u)) s=((r s) u) s=(1 u) s=u s$ and hence $s \in C(R)$. By Proposition 7 we are done if we can show $s \in N_{l}(R) \cap N_{m}(R)$. To this end, take $v \in R$. Then $s(u v)=s((1 u) v)=s(((r s) u) v)=(r s)((s u) v)=1((s u) v)=(s u) v$ which shows that $s \in N_{l}(R)$. We also see that $(u s) v=(u s)(1 v)=(u s)((r s) v)=(u(r s))(s v)=(u 1)(s v)=u(s v)$ which shows that $s \in N_{m}(R)$.

Proposition 9. If $R$ is simple, then $Z(R)$ is a field.

Proof. We already know that $Z(R)$ is a unital commutative ring. What is left to show is that every non-zero element of $Z(R)$ has a multiplicative inverse in $Z(R)$. To this end, take a non-zero $r \in Z(R)$. Then $R r$ is a non-zero ideal of $R$. Since $R$ is simple, this implies that $R=R r$. In particular, we get that there is $s \in R$ such that $1=s r$. By Proposition 8, we get that $s \in Z(R)$ and we are done.

\section{Non-Associative Ore EXtensions}

In this section, we show that there is a bijection between the set of (strong) non-associative Ore extensions of $R$ and the set of generalized polynomial rings $R[X ; \sigma, \delta]$ over $R$, where $\sigma$ (is a fixed point homomorphism) and $\delta$ (is a $\sigma$-kernel derivation) are additive maps $R \rightarrow R$ such that $\sigma(1)=1$ and $\delta(1)=0$ (see Proposition 14 and Proposition [15). We also show that if $S=R[X ; \sigma, \delta]$ is a generalized polynomial ring, then $S$ is associative if and only if $R$ is associative, $\sigma$ is a ring endomorphism and $\delta$ is a $\sigma$-derivation (see Proposition 17).

Throughout this section, $R$ denotes a non-associative ring.

Definition 10. By a formal set of polynomials $R[X]$ over $R$ we mean the collection of functions $f: \mathbb{N} \rightarrow R$ with the property that $f(n)=0$ for all but finitely many $n \in \mathbb{N}$. If $f, g \in R[X]$ and $r, s \in R$, then we define $r f+s g \in R[X]$ by the relation $(r f+s g)(n)=r f(n)+s g(n)$, for $n \in \mathbb{N}$. If we for each $n \in \mathbb{N}$, let $X^{n} \in R[X]$ be defined by $X^{n}(m)=1$, if $m=n$, and 
$X^{n}(m)=0$, if $m \neq n$, then $R[X]$ is a free left $R$-module with $B=\left\{X^{n}\right\}_{n \in \mathbb{N}}$ as a basis. In fact, for each $f \in R[X]$, we have that $f=\sum_{n \in \mathbb{N}} f(n) X^{n}$. By the degree of $f$, denoted by $\operatorname{deg}(f)$, we mean the supremum of $\{-\infty\} \cup\{n \in \mathbb{N} \mid f(n) \neq 0\}$. If $f \neq 0$, then we call $f(\operatorname{deg}(f))$ the leading coefficient of $f$. If the leading coefficient of $f$ is 1 , then we say that $f$ is monic.

Definition 11. Let $\sigma: R \rightarrow R$ and $\delta: R \rightarrow R$ be additive maps such that $\sigma(1)=1$ and $\delta(1)=0$. By the generalized polynomial ring $R[X ; \sigma, \delta]$ over $R$ defined by $\sigma$ and $\delta$ we mean the set $R[X]$ of formal polynomials over $R$ equipped with the product defined on monomials by the relation (1). We will often identify each $r \in R$ with $r X^{0}$. It is clear that $R[X ; \sigma, \delta]$ is a non-associative ring with $1=X^{0}$. It is also clear that $X$ is power associative so that $X^{n}$, for $n>0$, is in fact equal to the product of $X$ with itself $n$ times.

Definition 12. Suppose that $(S, x)$ is a non-associative Ore extension of $R$. Put $R_{x}=\{a \in$ $R \mid a x=x a\}$. We say that $(S, x)$ is strong if at least one of the following axioms holds:

(N4) $\left(x, R, R_{x}\right)=\{0\}$;

(N5) $\left(x, R_{x}, R\right)=\{0\}$.

In that case we call $R_{x}$ the ring of constants of $R$. If $(S, x)$ is a non-associative differential polynomial ring, then we say that it is strong if it is strong as a non-associative Ore extension.

The usage of the term "ring" in Definition 12 is justified by the next result.

Proposition 13. If $(S, x)$ is a strong non-associative Ore extensions of $R$, then $R_{x}$ is a subring of $R$.

Proof. It is clear that $R_{x}$ is an additive subgroup of $R$ containing 1 . Now we show that $R_{x}$ is multiplicatively closed. Take $a, b \in R_{x}$. Then $(a b) x \stackrel{(N 3)}{=} a(b x) \stackrel{\left[b \in R_{x}\right]}{=} a(x b) \stackrel{(N 3)}{=}(a x) b \stackrel{\left[a \in R_{x}\right]}{=}$ $(x a) b=x(a b)$. The last equality follows from the strongness of $(S, x)$. Therefore $a b \in R_{x}$.

Proposition 14. Every generalized polynomial ring $S=R[X ; \sigma, \delta]$ over $R$ (with $\sigma$ a fixed point homomorphism and $\delta$ a $\sigma$-kernel derivation) is a (strong) non-associative Ore extension of $R$ with $x=X$.

Proof. We first show the "non-strong" statement. From Definition 10, we know that $S$ is free as a left $R$-module with $B$ as a basis. Therefore (N1) holds. Also $R x=R X^{0} \cdot 1 X=$ $\delta(R)+\sigma(R) X=\delta(R)+\sigma(R) x \subseteq R+R x$. Therefore (N2) holds. Now we show (N3). Suppose that $a, b \in R$ and $m, n \in \mathbb{N}$. Then we get that $\left(a X^{m} \cdot b X^{n}\right) \cdot X=\sum_{i \in \mathbb{N}} a \pi_{i}^{m}(b) X^{i+n} \cdot X=$ $\sum_{i \in \mathbb{N}} a \pi_{i}^{m}(b) X^{i+n+1}=a X^{m} \cdot\left(b X^{n+1}\right)=a X^{m} \cdot\left(b X^{n} \cdot X\right)$. Next we get that $\left(a X^{m} \cdot X\right) \cdot b X^{n}=$ $a X^{m+1} \cdot b X^{n}=\sum_{i \in \mathbb{N}} a \pi_{i}^{m+1}(b) X^{i+n}=\sum_{i \in \mathbb{N}} a \pi_{i}^{m}(\delta(b)) X^{i+n}+\sum_{i \in \mathbb{N}} a \pi_{i-1}^{m}(\sigma(b)) X^{i+n}=$ $a X^{m} \cdot\left(\delta(b) X^{n}+\sigma(b) X^{n+1}\right)=a X^{m} \cdot\left(X \cdot b X^{n}\right)$. Now we show the "strong" statement. Note that $R_{X}=R_{\delta}^{\sigma}$. Suppose first that both $\sigma$ and $\delta$ are right $R_{\delta}^{\sigma}$-linear. We show (N4). To this end, take $a \in R$ and $b \in R_{X}$. Then $(X \cdot a) \cdot b=(\delta(a)+\sigma(a) X) \cdot b \stackrel{(N 3)}{=} \delta(a) b+\sigma(a)(X b)=[b \in$ $\left.R_{\delta}^{\sigma}\right]=\delta(a) b+\sigma(a)(b X) \stackrel{(N 3)}{=} \delta(a) b+(\sigma(a) b) X$. Since $\sigma$ and $\delta$ are right $R_{\delta}^{\sigma}$-linear, we get that $(X \cdot a) \cdot b=\delta(a b)+\sigma(a b) X=X \cdot(a b)$. Suppose now that both $\sigma$ and $\delta$ are left $R_{\delta}^{\sigma}$-linear. We show (N5). To this end, take $a \in R_{X}$ and $b \in R$. Then $(X \cdot a) \cdot b=\left[a \in R_{\delta}^{\sigma}\right]=(a \cdot X) \cdot b \stackrel{(N 3)}{=}$ $a \cdot(X b)=a \cdot(\delta(b)+\sigma(b) X)=a \delta(b)+a \sigma(b) X$. Since $\sigma$ and $\delta$ are left $R_{\delta}^{\sigma}$-linear, we get that $(X \cdot a) \cdot b=\delta(a b)+\sigma(a b) X=X \cdot(a b)$.

Proposition 15. Every non-associative Ore extension of $R$ is isomorphic to a generalized polynomial ring $R[X ; \sigma, \delta]$. If the non-associative Ore extension is strong, then $\sigma$ is a fixed point homomorphism and $\delta$ is a $\sigma$-kernel derivation. 
Proof. We first show the "non-strong" statement. Suppose that $S$ is a non-associative Ore extension of $R$ defined by the element $x \in S$. Take $a, b \in R$. By (N1) and (N2), we get that $x a=\delta(a)+\sigma(a) x$, for some unique $\delta(a), \sigma(a) \in R$. Hence this defines functions $\sigma: R \rightarrow R$ and $\delta: R \rightarrow R$. By distributivity of $S$, we get the relation $x(a+b)=x a+x b$ which implies that $\sigma(a+b)=\sigma(a)+\sigma(b)$ and $\delta(a+b)=\delta(a)+\delta(b)$. From the relation $x 1=x$ we get that $\sigma(1)=1$ and $\delta(1)=0$. Define $f: S \rightarrow R[X ; \sigma, \delta]$ by the additive extension of the relations $f\left(a x^{m}\right)=a X^{m}$, for $a \in R$ and $m \in \mathbb{N}$. Then clearly $f$ is an isomorphism of additive groups. What is left to show is that $f$ respects multiplication. Take $a, b \in R$ and $m, n \in \mathbb{N}$. We claim that $\left(a x^{m}\right)\left(b x^{n}\right)=\sum_{i \in \mathbb{N}} a \pi_{i}^{m}(b) x^{i+n}$. If we assume that the claim holds, then $f\left(\left(a x^{m}\right)\left(b x^{n}\right)\right)=f\left(\sum_{i \in \mathbb{N}} a \pi_{i}^{m}(b) x^{i+n}\right)=\sum_{i \in \mathbb{N}} a \pi_{i}^{m}(b) X^{i+n}=\left(a X^{m}\right) \cdot\left(b X^{n}\right)=$ $f\left(a x^{n}\right) \cdot f\left(b x^{m}\right)$. Now we prove the claim by induction over $m$.

First we show the base case $m=0$. By (N2) we get that $x \in N_{r}(S)$. Therefore $x^{n} \in N_{r}(S)$ and hence we get that $\left(a x^{0}\right)\left(b x^{n}\right)=a\left(b x^{n}\right)=(a b) x^{n}=a \pi_{0}^{0}(b) x^{n}=\sum_{i \in \mathbb{N}} a \pi_{i}^{0}(b) x^{i+n}$.

Next we show the induction step. Suppose that the claim holds for some $m \in \mathbb{N}$. By (N2), we get that $x \in N_{m}(S) \cap N_{r}(S)$. Therefore all powers of $x$ also belong to $N_{m}(S) \cap N_{r}(S)$ and hence we get that $\left(a x^{m+1}\right)\left(b x^{n}\right)=\left(a\left(x^{m} x\right)\right)\left(b x^{n}\right)=\left(\left(a x^{m}\right) x\right)\left(b x^{n}\right)=\left(a x^{m}\right)\left(x\left(b x^{n}\right)\right)=$ $\left(a x^{m}\right)\left((x b) x^{n}\right)=\left(a x^{m}\right)\left((\delta(b)+\sigma(b) x) x^{n}\right)=\left(a x^{m}\right)\left(\delta(b) x^{n}+\sigma(b) x^{n+1}\right)=\left(a x^{m}\right)\left(\delta(b) x^{n}\right)+$ $\left(a x^{m}\right)\left(\sigma(b) x^{n+1}\right)$. By the induction hypothesis the last expression equals $\sum_{i \in \mathbb{N}} a \pi_{i}^{m}(\delta(b)) x^{i+n}+$ $\sum_{i \in \mathbb{N}} a \pi_{i}^{m}(\sigma(b)) x^{i+n+1}=\sum_{i \in \mathbb{N}} a \pi_{i}^{m}(\delta(b)) x^{i+n}+\sum_{i \in \mathbb{N}} a \pi_{i-1}^{m}(\sigma(b)) x^{i+n}=\sum_{i \in \mathbb{N}} a\left[\pi_{i}^{m}(\delta(b))+\right.$ $\left.\pi_{i-1}^{m}(\sigma(b))\right] x^{i+n}=\sum_{i \in \mathbb{N}} a \pi_{i}^{m+1}(b) x^{i+n}$. This proves the induction step. Now we show the "strong" statement. To this end, take $a \in R_{x}$ and $b \in R$. Suppose first that (N5) holds. Then $x(a b)=(x a) b$. Thus, since $a \in R_{x}$, we get that $\delta(a b)+\sigma(a b) x=(a x) b \stackrel{(N 3)}{=} a(x b)=$ $a(\delta(b)+\sigma(b) x)=a \delta(b)+a(\sigma(b) x) \stackrel{(N 3)}{=} a \delta(b)+(a \sigma(b)) x$. Hence by (N1), we get that $\delta(a b)=a \delta(b)$ and $\sigma(a b)=a \sigma(b)$. Suppose now that (N4) holds. Then $x(b a)=(x b) a$. Thus $\delta(b a)+\sigma(b a) x=(\delta(b)+\sigma(b) x) a=\delta(b) a+(\sigma(b) x) \stackrel{(N 3)}{=} \delta(b) a+\sigma(b)(x a)=[a \in$ $\left.R_{x}\right]=\delta(b) a+\sigma(b)(a x) \stackrel{(N 3)}{=} \delta(b) a+(\sigma(b) a) x$. Hence, by (N1), we get that $\delta(b a)=\delta(b) a$ and $\sigma(b a)=\sigma(b) a$. Thus, in either case, $\sigma$ is a fixed point homomorphism of $R$ and $\delta$ is a $\sigma$-kernel derivation of $R$.

For use in later sections, we now note that the axioms (N4) and (N5) of Definition 12 can be replaced by seemingly stronger statements.

Proposition 16. Let $(S, x)$ be a non-associative Ore extension of $R$.

(a) The axiom (N4) holds if and only if $\left(\mathbb{Z}[x], S, R_{x}[x]\right)=\{0\}$ holds.

(b) The axiom (N5) holds if and only if $\left(\mathbb{Z}[x], R_{x}[x], S\right)=\{0\}$ holds.

Proof. Since the "if" statements are trivial, we only show the "only if" statements. To this end, take $a \in R_{x}, b \in R$ and $m, n, p \in \mathbb{N}$.

(a) We need to show that $\left(x^{n}, b x^{m}, a x^{p}\right)=0$. Since $x \in N_{m}(S) \cap N_{r}(S)$ and $a \in R_{x}$ it is enough to show this relation for $m=p=0$. Since (N4) holds, we get, from the proof of Proposition 15, that $\left(x^{n} b\right) a=\sum_{i \in \mathbb{N}} \pi_{i}^{n}(b) x^{i} a=\sum_{i \in \mathbb{N}} \pi_{i}^{n}(b) a x^{i}=\sum_{i \in \mathbb{N}} \pi_{i}^{n}(b a) x^{i}=x^{n}(b a)$.

(b) We need to show that $\left(x^{n}, a x^{p}, b x^{m}\right)=0$. Since $x \in N_{m}(S) \cap N_{r}(S)$ and $a \in R_{x}$ it is enough to show this relation for $m=p=0$. Since (N5) holds, we get, from the proof of Proposition [15, that $\left(x^{n} a\right) b=\left(a x^{n}\right) b=a\left(x^{n} b\right)=\sum_{i \in \mathbb{N}} a \pi_{i}^{n}(b) x^{i}=\sum_{i \in \mathbb{N}} \pi_{i}^{n}(a b) x^{i}=$ $x^{n}(a b)$.

Proposition 17. If $S=R[X ; \sigma, \delta]$ is a generalized polynomial ring, then 
(a) $R \subseteq N_{l}(S)$ if and only if $R$ is associative;

(b) $X \in N_{l}(S)$ if and only if $\sigma$ is a ring endomorphism and $\delta$ is a $\sigma$-derivation;

(c) $S$ is associative if and only if $R$ is associative, $\sigma$ is a ring endomorphism and $\delta$ is a $\sigma$-derivation.

Proof. (a) The "only if" statement is clear. Now we show the "if" statement. Suppose that $R$ is associative. Take $a, b, c \in R$ and $m, n \in \mathbb{N}$. We wish to show that

$$
\left(a, b X^{m}, c X^{n}\right)=0 .
$$

Since $X \in N_{r}(S)$, we get that $\left(a, b X^{m}, c X^{n}\right)=\left(a, b X^{m}, c\right) X^{n}$. Thus it is enough to prove (5) for $n=0$. Since $X \in N_{m}(S) \cap N_{r}(S)$ we get that $\left(a, b X^{m}, c\right)=\left(a, b, X^{m} c\right)=\sum_{i \in \mathbb{N}}\left(a, b, \pi_{i}^{m}(c) X^{i}\right)=$ $\sum_{i \in \mathbb{N}}\left(a, b, \pi_{i}^{m}(c)\right) X^{i}=0$, using that $R$ is associative.

(b) First we show the "only if" statement. Suppose that $X \in N_{l}(S)$. Take $a, b \in R$. From the equality $X(a b)=(X a) b$ we get that $\delta(a b)+\sigma(a b) X=(\delta(a)+\sigma(a) X) b \stackrel{(N 3)}{=} \delta(a) b+\sigma(a)(X b)=$ $\delta(a) b+\sigma(a)(\delta(b)+\sigma(b) X) \stackrel{(N 3)}{=} \delta(a) b+\sigma(a) \delta(b)+(\sigma(a) \sigma(b)) X$. Hence, by (N1), we get that $\sigma$ is a homomorphism and $\delta$ is a $\sigma$-derivation. Now we show the "if" statement. Suppose that $\sigma$ is a homomorphism and that $\delta$ is a $\sigma$-derivation. From the calculation in the proof of the "only if" statement it follows that $X \in N_{l}(R)$. From the same type of reasoning that we used in the proof of the "if" statement in (a), we therefore get that $(X, S, S) \subseteq \sum_{i \in \mathbb{N}}(X, R, R) X^{i}=\{0\}$.

(c) The "only if" statement follows directly from (a) and (b). Now we show the "if" statement. Suppose that $R$ is associative, $\sigma$ is a ring endomorphism and that $\delta$ is a $\sigma$-derivation. Take $a \in R$ and $m \in \mathbb{N}$. From (a) and (b) we get that $a, X \in N_{l}(S)$. Since $N_{l}(S)$ is multiplicatively closed we get that $a X^{m} \in N_{l}(S)$. Since $N_{l}(S)$ is closed under addition, we get that $S \subseteq N_{l}(S)$ and thus $S$ is associative.

Proposition 18. If $S=R[X ; \sigma, \delta]$ is a generalized polynomial ring with $\sigma$ bijective, then $B=\left\{X^{n}\right\}_{n \in \mathbb{N}}$ is a basis for $S$ as a right $R$-module.

Proof. First we show that $B$ is a right $R$-linearly independent set. We will show that for each $n \in \mathbb{N}$, the set $B_{n}:=\left\{X^{i}\right\}_{i=0}^{n}$ is right $R$-linearly independent. We will prove this by induction over $n$. Base case: $n=0$. It is clear that $\{1\}$ is right $R$-linearly independent. Induction step: suppose that $B_{n}$ is right $R$-linearly independent for some $n \in \mathbb{N}$. Suppose that $a_{i} \in R$, for $i \in$ $\{1, \ldots, n+1\}$, are chosen so that $\sum_{i=0}^{n+1} X^{i} a_{i}=0$. Then $0=\sigma^{n+1}\left(a_{n+1}\right) X^{n+1}+$ [lower terms]. Since $B_{n+1}$ is left $R$-linearly independent, we get that $\sigma^{n+1}\left(a_{n+1}\right)=0$. Since $\sigma$ is injective, we get that $a_{n+1}=0$. Thus $\sum_{i=0}^{n} X^{i} a_{i}=0$. By the induction hypothesis, we get that $a_{i}=0$, for $i \in\{0, \ldots, n\}$.

Next we show that $B$ right $R$-spans $S$. For each $n \in \mathbb{N}$, let $S_{n}$ (or $T_{n}$ ) denote the left (or right) $R$-span of $B_{n}$. We will show that for each $n \in \mathbb{N}$, the relation $S_{n}=T_{n}$ holds. We will prove this by induction over $n$. Base case: $n=0$. It is clear that $S_{0}=R=T_{0}$. Induction step: suppose that $S_{n}=T_{n}$ for some $n \in \mathbb{N}$. Take $a=\sum_{i=0}^{n+1} a_{i} X^{i} \in S_{n+1}$. Since $\sigma$ is surjective, we can pick $r \in R$ such that $\sigma^{n+1}(r)=a_{n+1}$. This implies that $a-X^{n+1} r \in S_{n}$. By the induction hypothesis this implies that $a-X^{n+1} r \in T_{n}$. Thus $a \in T_{n}+X^{n+1} r \subseteq T_{n+1}$. Thus $S_{n+1} \subseteq T_{n+1}$. Since the inclusion $S_{n+1} \supseteq T_{n+1}$ trivially holds, the induction step is complete.

Explicit formulas for how elements of generalized polynomial rings can be expressed as right $R$-linear combinations of elements from $B$ can be worked out exactly as in the classical case 
(see e.g. the formulas right after Theorem 7 in Ore's classical article 23]). In this article, we only need the following special case of these relations.

Proposition 19. Suppose that $S=R\left[X ; \mathrm{id}_{R}, \delta\right]$ is a non-associative differential polynomial ring. If $r \in R$ and $n \in \mathbb{N}$, then $r X^{n}=\sum_{i=0}^{n}(-1)^{i}\left(\begin{array}{c}n \\ i\end{array}\right) X^{n-i} \delta^{i}(r)$.

Proof. We will show this by induction over $n$. Base case: $n=0$. This is clear since $r X^{0}=$ $r=X^{0} r$. Induction step: suppose that $r X^{n}=\sum_{i=0}^{n}(-1)^{i}\left(\begin{array}{c}n \\ i\end{array}\right) X^{n-i} \delta^{i}(r)$ for some $n \in \mathbb{N}$. Then, since $X \in N_{m}(S) \cap N_{r}(S)$, we get that $r X^{n+1}=r X^{n} X=\sum_{i=0}^{n}(-1)^{i}\left(\begin{array}{c}n \\ i\end{array}\right) X^{n-i} \delta^{i}(r) X=$ $\sum_{i=0}^{n}(-1)^{i}\left(\begin{array}{c}n \\ i\end{array}\right) X^{n-i}\left(X \delta^{i}(r)-\delta^{i+1}(r)\right)=\sum_{i=0}^{n}(-1)^{i}\left(\begin{array}{c}n \\ i\end{array}\right) X^{n+1-i} \delta^{i}(r)+(-1)^{i+1}\left(\begin{array}{c}n \\ i\end{array}\right) X^{n-i} \delta^{i+1}(r)=$ $\left[\left(\begin{array}{c}n+1 \\ i\end{array}\right)=\left(\begin{array}{c}n \\ i\end{array}\right)+\left(\begin{array}{c}n \\ i-1\end{array}\right)\right]=\sum_{i=0}^{n+1}(-1)^{i}\left(\begin{array}{c}n+1 \\ i\end{array}\right) X^{n+1-i} \delta^{i}(r)$

\section{Ideal Structure}

The aim of this section is to prove Theorem 6. To this end, we first show a series of results concerning simplicity and the center. Throughout this section, $R$ denotes a non-associative ring and $\sigma$ and $\delta$ are additive maps $R \rightarrow R$ satisfying $\sigma(1)=1$ and $\delta(1)=0$. Furthermore, we let $S=R[X ; \sigma, \delta]$ denote a non-associative Ore extension of $R$.

Definition 20. An ideal $I$ of $R$ is said to be $\sigma$ - $\delta$-invariant if $\sigma(I) \subseteq I$ and $\delta(I) \subseteq I$. If $\{0\}$ and $R$ are the only $\sigma$ - $\delta$-invariant ideals of $R$, then $R$ is said to be $\sigma-\delta$-simple.

Proposition 21. If $S$ is simple, then $R$ is $\sigma-\delta$-simple.

Proof. Take a non-zero $\sigma$ - $\delta$-invariant ideal $J$ of $R$. We wish to show that $J=R$. Let $I=\oplus_{i \in \mathbb{N}} J X^{i}$. Since $J$ is a right ideal of $R$ it follows that $I$ is a right ideal of $S$. Using that $J$ is $\sigma$ - $\delta$-invariant it follows that $I$ is a left ideal of $S$. Since $J$ is non-zero it follows that $I$ is non-zero. By simplicity of $S$, we get that $I=S$ and thus $J=R$.

Proposition 22. Suppose that $R$ is $\sigma$ - $\delta$-simple. If $\sigma$ is a fixed point homomorphism and $\delta$ is a $\sigma$-kernel derivation, then $Z(R)_{\delta}^{\sigma}$ is a field.

Proof. Put $T=Z(R)_{\delta}^{\sigma}$. We already know that $Z(R)$ is an associative commutative unital ring. Suppose that $\sigma$ and $\delta$ are right $R_{\delta}^{\sigma}$-linear. Take $a, b \in T$. We have $\sigma(a b)=\sigma(a) b=a b$ and $\delta(a b)=\delta(a) b=0 b=0$. Thus $a b \in T$. Since it is clear that $1 \in T$ and that $T$ is additively closed, it follows that $T$ is an associative commutative unital ring. What remains to show is that every non-zero element of $T$ has a multiplicative inverse. To this end, take a non-zero $a \in T$. Then $R a$ is a non-zero ideal of $R$ with $\sigma(R a)=\sigma(R) a \subseteq R a$ and $\delta(R a)=\delta(R) a \subseteq R a$. Hence $R a$ is $\sigma$ - $\delta$-invariant. By $\sigma-\delta$-simplicity of $R$, we get that $R a=R$. Thus, there is $b \in R$ such that $a b=1$. By Proposition [8, we get that $b \in Z(R)$. Now we show that $b \in R_{\delta}^{\sigma}$. Indeed, $\sigma(b)=\sigma(b) 1=\sigma(b) a b=\sigma(b a) b=\sigma(1) b=1 b=b$ and $\delta(b)=\delta(b) 1=\delta(b) a b=\delta(b a) b=\delta(1) b=0 b=0$. This shows that $b \in T$. The left $R_{\delta}^{\sigma}$-linear case is treated analogously.

Proposition 23. If $a \in R_{\delta}^{\sigma}[X]$ commutes with every element of $R$, then $a \in C(S)$.

Proof. First we show, using induction, that, for every $n \in \mathbb{N}$, the relation $\left[a, x^{n}\right]=0$ holds. The base case $n=0$ follows immediately since $\left[a, X^{0}\right]=[a, 1]=0$. Now we show the induction step. Suppose that $\left[a, X^{n}\right]=0$ for some $n \in \mathbb{N}$. Then $\left[a, X^{n+1}\right]=a X^{n+1}-X^{n+1} a=a\left(X X^{n}\right)-$ $\left(X X^{n}\right) a=(a X) X^{n}-X\left(X^{n} a\right)=(a X) X^{n}-X\left(a X^{n}\right)=(a X) X^{n}-(X a) X^{n}=[a, X] X^{n}=0$, since it follows from $a \in R_{\delta}^{\sigma}[X]$ that $[a, X]=0$. Now $\left[a, b X^{n}\right]=a\left(b X^{n}\right)-\left(b X^{n}\right) a=(a b) X^{n}-$ $b\left(X^{n} a\right)=(a b) X^{n}-b\left(a X^{n}\right)=(a b) X^{n}-(b a) X^{n}=[a, b] X^{n}=0$. 
Proposition 24. Suppose that $S$ is a strong non-associative Ore extension of $R$. If a $\in R_{\delta}^{\sigma}[X]$ commutes with every element of $R$, and associates with all elements of $R$, then $a \in Z(S)$.

Proof. By Proposition 23 we conclude that $a \in C(S)$. Since $Z(S)=C(S) \cap N(S)$, we need to show that $a \in N(S)$. First we show that $a \in N_{l}(S)$. Take $n, p \in \mathbb{N}$. Since $(a, R, R)=\{0\}$ and $X \in N_{m}(S) \cap N_{r}(S)$, we get that $\left(a, R X^{n}, R X^{p}\right)=\left(a, R X^{n}, R\right) X^{p}=\left(a, R, X^{n} R\right) X^{p} \subseteq$ $\sum_{i \in \mathbb{N}}\left(a, R, \pi_{i}^{n}(R) X^{i}\right) X^{p} \subseteq \sum_{i=1}^{n}(a, R, R) X^{i+p}=\{0\}$. By Proposition 7, we are done if we can show that $a \in N_{m}(S)$ or $a \in N_{r}(S)$.

Case 1: (N4) holds. We show that $a \in N_{r}(S)$. We wish to show that

$$
\left(b X^{n}, c X^{p}, a\right)=0 .
$$

Since $X \in N_{m}(S) \cap N_{r}(S)$ and $a \in C(S)$, we get that $\left(\left(b X^{n}\right)\left(c X^{p}\right)\right) a=\left(\left(\left(b X^{n}\right) c\right) X^{p}\right) a=$ $\left(\left(b X^{n}\right) c\right)\left(X^{p} a\right)=\left(\left(b X^{n}\right) c\right)\left(a X^{p}\right)=\left(\left(\left(b X^{n}\right) c\right) a\right) X^{p}=\left(\left(b\left(X^{n} c\right)\right) a\right) X^{p}$ and, by Proposition 16(a), we get that $b X^{n}\left(\left(c X^{p}\right) a\right)=b X^{n}\left(c\left(X^{p} a\right)\right)=b X^{n}\left(c\left(a X^{p}\right)\right)=b X^{n}\left((c a) X^{p}\right)=\left(b X^{n}(c a)\right) X^{p}=$ $\left(b\left(X^{n}(c a)\right)\right) X^{p}=\left(b\left(\left(X^{n} c\right) a\right)\right) X^{p}$. This shows (6) $)$.

Case 2: (N5) holds. We show that $a \in N_{m}(S)$. We wish to show that

$$
\left(b X^{n}, a, c X^{p}\right)=0 .
$$

Since $X \in N_{r}(S)$, we only need to show (7) for $p=0$. Since $X \in N_{m}(S) \cap N_{r}(S)$, $a \in C(S)$ and $a$ associates with all elements of $R$, we get that $\left(\left(b X^{n}\right) a\right) c=\left(b\left(X^{n} a\right)\right) c=$ $\left(b\left(a X^{n}\right)\right) c=\left((b a) X^{n}\right) c=(b a)\left(X^{n} c\right)=\sum_{i \in \mathbb{N}}(b a) \pi_{i}^{n}(c) X^{i}=\sum_{i \in \mathbb{N}} b\left(a \pi_{i}^{n}(c)\right) X^{i}$. On the other hand, since $a \in C(S), X \in N_{m}(S) \cap N_{r}(S)$ and Proposition 16)(b) holds, we get that $b X^{n}(a c)=b\left(X^{n}(a c)\right)=b\left(\left(X^{n} a\right) c\right)=b\left(\left(a X^{n}\right) c\right)=b\left(a\left(X^{n} c\right)\right)=\sum_{i \in \mathbb{N}} b\left(a \pi_{i}^{n}(c)\right) X^{i}$. This shows (7).

Corollary 25. If $\delta$ is a kernel derivation on $R$ and we put $D=R\left[X ; \operatorname{id}_{R}, \delta\right]$, then $Z(D)$ is the set of all $a \in D$ such that (i) a commutes with $X$, and (ii) a commutes with all elements of $R$, and (iii) a associates with all elements of $R$.

Proposition 26. Let $\sigma$ be injective and suppose that $a, b \in S=R[X ; \sigma, \delta]$ are elements such that $a b=b a=1$. If the leading coefficient of $a$ is a regular element of $R$, then $a, b \in R$.

Proof. Suppose that $b=\sum_{i=0}^{m} b_{i} X^{i}$, where $b_{m} \neq 0$. Comparing coefficients of $X^{n+m}$ in the relation $a b=1$ we get that $a_{n} \sigma^{n}\left(b_{m}\right)=0$ if $m+n>0$. Since $a_{n}$ is regular, we therefore get that $\sigma^{n}\left(b_{m}\right)=0$ whenever $m+n>0$. By injectivity of $\sigma$, we get $b_{m}=0$ if $m>0$. Comparing coefficients of degree $n$ in the relation $b a=b_{0} a=1$ we get that $b_{0} a_{n}=0$ if $n>0$. Since $b_{0}=b \neq 0$ and $a_{n}$ is regular, we get that $n=0$. Hence $m=n=0$ and $a, b \in R$.

Proposition 27. If $a, b \in S$, then $\operatorname{deg}(a b) \leq \operatorname{deg}(a)+\operatorname{deg}(b)$. Moreover, if $b$ is monic or a is monic and $\sigma$ is injective, then equality holds.

Proof. Suppose that $\operatorname{deg}(a)=m$ and $\operatorname{deg}(b)=n$. Let $a_{m}$ and $b_{n}$ denote the leading coefficients of $a$ and $b$ respectively. Then $a b=a_{m} \sigma^{m}\left(b_{n}\right) X^{m+n}+$ [lower terms]. So $\operatorname{deg}(a b) \leq m+n=$ $\operatorname{deg}(a)+\operatorname{deg}(b)$. Equality holds if and only if $a_{m} \sigma^{m}\left(b_{n}\right) \neq 0$. This holds in particular if $b_{n}=1$ or if $a_{m}=1$ and $\sigma$ is injective.

Next we show that there in some cases is a Euclidean algorithm for $S$.

Proposition 28. If $a, b \in S$ where $b$ is monic, then $a=q b+r$ for suitable $q, r \in S$ such that either $r=0$ or $\operatorname{deg}(r)<\operatorname{deg}(b)$. 
Proof. We follow closely the proof in [27, p. 94] for the associative case. Without loss of generality, we may assume that $a \neq 0$. Suppose that $\operatorname{deg}(a)=m$ and $\operatorname{deg}(b)=n$. Let $a_{m}$ denote the leading coefficient of $a$. Case 1: $m<n$. Then we can put $q=0$ and $r=a$. Case 2: $m \geq n$. Put $c=a-\left(a_{m} X^{m-n}\right) b$. Then $\operatorname{deg}(c)<\operatorname{deg}(a)$. By induction there are $q^{\prime}, r^{\prime} \in S$ with $c=q^{\prime} b+r^{\prime}$ and $r^{\prime}=0$ or $\operatorname{deg}\left(r^{\prime}\right)<n$. This implies that $a=\left(a_{m} X^{m-n}\right) b+c=$ $\left(a_{m} X^{m-n}\right) b+q^{\prime} b+r^{\prime}=\left(a_{m} X^{m-n}+q^{\prime}\right) b+r^{\prime}$. So we can put $q=a_{m} X^{m-n}+q^{\prime}$ and $r=r^{\prime}$.

\section{Proof of Theorem 6.}

Proof of (a). Let $I$ be an ideal of $D$. Suppose that $m$ is the minimal degree of non-zero elements of $I$. Put $J=\left\{r \in R \mid \exists r_{0}, r_{1}, \ldots, r_{m-1} \in R: r X^{m}+r_{m-1} X^{m-1}+\ldots+r_{0} \in I\right\}$. It is clear that $J$ is a ideal of $R$. From the fact that $X I-I X \subseteq I$ it follows that $J$ is $\delta$-invariant. Since $R$ is $\delta$-simple and $J$ is non-zero, we can conclude that $J=R$. In particular, $1 \in J$. Therefore there is a monic $a \in I$ of degree $m$.

Now we show that $a \in Z(D)$. To this end, we check (i), (ii) and (iii) of Corollary 25. Since $a \in D_{\delta}$, (i) holds. Now we check (ii). Take $r \in R$. Since $a$ is monic the leading coefficient of $[a, r]$ is $[1, r]=0$. Thus $\operatorname{deg}([a, r])<m$ which, since $[a, r] \in I$, implies that $[a, r]=0$, by minimality of $m$. Now we check (iii). Take $r, s \in R$. Since $a$ is monic and the leading coefficients of all the polynomials $(a, r, s),(r, a, s)$ and $(r, s, a)$ equal zero, all of them have degree less that $m$. By minimality of $m$ and the fact that all of these polynomials belong to $I$, we get that they are zero. Thus (iii) holds.

Next we show that $I=D a$. The inclusion $I \supseteq D a$ is clear. Now we show the reversed inclusion. Take a non-zero $c \in I$. Since $\operatorname{deg}(c) \geq \operatorname{deg}(a)$, we can use Proposition 28 to conclude that $c=q a+r$, for some $q, r \in S$ with $\operatorname{deg}(r)<\operatorname{deg}(a)$. But then $r=c-q a \in I$, which, by minimality of $m$, implies that $r=0$. Therefore $c=q a \in I$. Hence $I \subseteq D a$.

Finally we show uniqueness of $a$. Suppose that $d \in D$ is monic and $I=D d$. From the relations $a \in D d$ and $d \in D a$ we get, respectively from Proposition 27, that $\operatorname{deg}(a) \geq \operatorname{deg}(d)$ and $\operatorname{deg}(d) \geq \operatorname{deg}(a)$, which together imply that $\operatorname{deg}(a)=\operatorname{deg}(d)$. Since $a$ and $d$ are monic, we get that $\operatorname{deg}(a-d)<m$, which, by $a-d \in I$ and minimality of $m$, implies that $a=d$.

Proof of (b). Case 1: $Z(D)$ only contains polynomials of degree zero. Then $Z(D) \subseteq Z(R)_{\delta}$. But since $Z(R)_{\delta} \subseteq Z(D)$ we get that $Z(D)=Z(R)_{\delta}$ and we can choose $b=1$.

Case 2: $Z(D)$ contains polynomials of degree greater than zero. Let $n$ denote the least degree of non-constant polynomials in $Z(D)$. Take $b \in Z(D)$ such that $\operatorname{deg}(b)=n$. Now we show that we may choose $b$ to be monic. Since $I=D b$ is an ideal of $D$, by (a), we may choose a monic $f \in I \cap Z(D)_{\delta}$ such that $I=D f$. But then $b=c f$ for some $c \in D$. Since $f$ is monic we get that $n=\operatorname{deg}(b)=\operatorname{deg}(c)+\operatorname{deg}(f)$ which implies that $\operatorname{deg}(f) \leq n$. By minimality of $n$ we get that $\operatorname{deg}(f)=n$ and we may choose $b$ to be the monic $f$.

Now take $g \in Z(D)$ of degree $m$. We will show by induction over the degree of $g$ that $g \in Z_{\delta}(R)[b]$. Base case: $m=0$, i.e. $g$ is constant. Then $g \in R \cap Z(S)=Z_{\delta}(R) \subseteq Z_{\delta}(R)[b]$. Induction step: suppose that $m>0$ and that we have shown the claim for all $m^{\prime}<m$. Since $b$ is monic, we can write $g=h b+k$ for some $h, k \in S$ with $\operatorname{deg}(k)<\operatorname{deg}(b)$. Note that, since $b$ is monic, we get that $\operatorname{deg}(h)<\operatorname{deg}(g)$. We claim that $h, k \in Z(D)$. If we assume that the claim holds, then, by the induction hypothesis, we are done. Now we show the claim. To this end, we will check (i),(ii) and (iii) in Corollary 25. First we check (i). Note that $0=[X, g]=[X, h] b+[X, k]$. Seeking a contradiction, suppose that $[X, h] \neq 0$. Since $b$ is monic and $\operatorname{deg}([X, k]) \leq \operatorname{deg}(k)$, we get the contradiction $-\infty=\operatorname{deg}(0)=\operatorname{deg}([X, g])=$ $\operatorname{deg}([X, h] b+[X, k]) \geq n$. Therefore $[X, h]=0$ and hence $[X, k]=0$. In other words $h, k \in$ 
$R_{\delta}[X]$. Now we show (ii). To this end, note that $0=[r, g]=[r, h] b+[r, k]$. Seeking a contradiction, suppose that $[r, h] \neq 0$. Since $b$ is monic and $\operatorname{deg}([r, k]) \leq \operatorname{deg}(k)$, we get the contradiction $-\infty=\operatorname{deg}(0)=\operatorname{deg}([r, g])=\operatorname{deg}([r, h] b+[r, k]) \geq n$. Therefore $[r, h]=0$ and hence $[r, k]=0$. Finally, we show (iii). Take $r, s \in R$. Let $\alpha(\cdot)$ denote either of the maps $(\cdot, r, s),(r, \cdot, s)$ or $(r, s, \cdot)$. Then $0=\alpha(g)=\alpha(h) b+\alpha(k)$. Seeking a contradiction, suppose that $\alpha(h) \neq 0$. Since $b$ is monic and $\operatorname{deg}(\alpha(k)) \leq \operatorname{deg}(k)$, we get the contradiction $-\infty=\operatorname{deg}(0)=\operatorname{deg}(\alpha(g))=\operatorname{deg}(\alpha(h) b+\alpha(k)) \geq n$. Therefore $\alpha(h)=0$ and hence $\alpha(k)=0$. This completes the induction step.

Now we show uniqueness of $b$ up to addition by an element from $Z(R)_{\delta}$. Case 1: $Z(D)$ only contains polynomials of degree zero. Then there is only one monic polynomial in $Z(D)$, namely $b=1$.

Case 2: $Z(D)$ contains polynomials of degree greater than zero i.e. $n>0$. Suppose that there is another monic $b^{\prime} \in R_{\delta}[X]$ such that $Z(D)=Z(R)_{\delta}\left[b^{\prime}\right]$. Then there is a polynomial $p \in Z(R)_{\delta}[X]$ such that $b=p\left(b^{\prime}\right)$. Hence $n=\operatorname{deg}(b)=\operatorname{deg}(p(b)) \geq \operatorname{deg}\left(b^{\prime}\right)$. By minimality of $n$, we get that $\operatorname{deg}\left(b^{\prime}\right)=n$. But then $b-b^{\prime}$ is a polynomial in $Z(D)$ of degree less than $n$, which, by minimality of $n$, implies that $b-b^{\prime} \in Z(R)_{\delta}$.

Proof of (c). First we show the "only if" statement. Suppose that $D$ is simple. By Proposition 21, we get that $R$ is $\delta$-simple. By Proposition 9, we get that $Z(D)$ is a field.

Next we show the "if" statement. Suppose that $R$ is $\delta$-simple and that $Z(D)$ is a field. Let $I$ be a non-zero ideal of $D$. By (a) and Proposition 26, this implies that the polynomial in $Z(D)$ corresponding to $I$ is 1 . This implies that $I=D$.

By (b) and Proposition [26, the ring $Z(R)_{\delta}[b]$ is a field precisely when $b=1$.

Proofs of (d) and (e). By Proposition [18, we can write $b=\sum_{i=0}^{n} b_{i} X^{i}$, where $b_{i} \in R$, for $i \in\{1, \ldots, n\}$, with $b_{n}=1$. Since $b \in Z(D)$, we get, in particular, that $X b=b X$. This implies that $\delta\left(b_{i}\right)=0$, for $i \in\{1, \ldots, n\}$. Therefore $b=\sum_{i=0}^{n} X^{i} b_{i}$. For every $j \in\{1, \ldots, n\}$ define the polynomial $c_{j}=\sum_{i=j}^{n} X^{i-j}\left(\begin{array}{l}i \\ j\end{array}\right) b_{i}$. We claim that each $c_{j} \in Z(D)$. If we assume that the claim holds, then, by minimality of $n$, we get that $b_{j}=c_{j} \in Z(R)_{\delta}$ and that $\left(\begin{array}{l}i \\ j\end{array}\right) b_{i}=0$ whenever $1 \leq j<i \leq n$. In the case when the characteristic of $Z(R)_{\delta}$ is zero, we therefore get that $b=1$ or $b=b_{0}+X$. The relation $b r=r b$ now gives us that $\delta=\delta_{b_{0}}$. Now suppose that the characteristic of $Z(R)_{\delta}$ is a prime $p$. Fix $i \in\{1, \ldots, n\}$ such that $b_{i}$ is non-zero. Then $\left(\begin{array}{l}i \\ j\end{array}\right)=0$ when $1 \leq j<i$. By Lucas' Theorem (see e.g. [9]) this implies that $i$ must be a power of $p$. Choose the smallest $q \in \mathbb{N}$ such that $p^{q} \leq n$. For each $i \in \mathbb{N}$ put $c_{i}=b_{p^{i}}$. Also put $c=b_{0}$. Then $b=c+\sum_{i=0}^{q} c_{i} \delta^{p^{i}}$. The relation $b r=r b$ now gives us that $\delta_{c}+\sum_{i=0}^{n} c_{i} \delta^{p^{i}}=0$.

Now we show the claim. To this end, we will check conditions (i), (ii) and (iii) of Corollary 25. Since $\delta\left(b_{i}\right)=0$ we know that (i) holds. Now we show (ii). Take $r \in R$. First note that since $b r=r b$, we can use Proposition 19 to conclude that

$$
b_{v} r=\sum_{i=v}^{n}(-1)^{i-v}\left(\begin{array}{c}
i \\
i-v
\end{array}\right) \delta^{i-v}(r) b_{i}
$$


for each $v \in\{0, \ldots, n\}$. Thus,

$$
\begin{aligned}
& c_{j} r=\sum_{i=j}^{n} r\left(X^{i-j}\left(\begin{array}{l}
i \\
j
\end{array}\right) b_{i}\right) \stackrel{\left[X \in N_{m}(D)\right]}{=} \sum_{i=j}^{n}\left(r X^{i-j}\right)\left(\begin{array}{l}
i \\
j
\end{array}\right) b_{i} \\
& =\sum_{i=j}^{n}\left(\sum_{k=0}^{i-j} X^{i-j-k}(-1)^{k}\left(\begin{array}{c}
i-j \\
k
\end{array}\right) \delta^{k}(r)\right)\left(\begin{array}{l}
i \\
j
\end{array}\right) b_{i} \stackrel{\left[X \in N_{l}(D)\right]}{=} \\
& =\sum_{i=j}^{n} \sum_{k=0}^{i-j} X^{i-j-k}(-1)^{k}\left(\begin{array}{c}
i-j \\
k
\end{array}\right) \delta^{k}(r)\left(\begin{array}{l}
i \\
j
\end{array}\right) b_{i} \stackrel{[v=i-k]}{=} \\
& =\sum_{i=v}^{n} \sum_{v=j}^{n} X^{v-j}\left(\begin{array}{l}
i \\
j
\end{array}\right)\left(\begin{array}{l}
i-j \\
i-v
\end{array}\right)(-1)^{i-v} \delta^{i-v}(r) b_{i} \\
& =\sum_{i=v}^{n} \sum_{v=j}^{n} X^{v-j}\left(\begin{array}{c}
v \\
j
\end{array}\right)\left(\begin{array}{c}
i \\
i-v
\end{array}\right)(-1)^{i-v} \delta^{i-v}(r) b_{i} \stackrel{[\text { Eq. [8] }]}{=} \sum_{v=j}^{n} X^{v-j}\left(\begin{array}{l}
v \\
j
\end{array}\right) b_{v} r=c_{j} r .
\end{aligned}
$$

Finally, we show (iii). Take $r, s \in R$. From the relations $(r, s, b)=0$ and $(b, r, s)=0$ it follows that $\left(r, s, b_{i}\right)=\left(b_{i}, r, s\right)=0$. Hence we get that $\left(r, s, c_{j}\right)=\left(c_{j}, r, s\right)=0$. Thus $c_{j} \in N_{r}(R) \cap N_{l}(R)$. Since $c_{j} \in C(R)$, we now automatically get that $\left(r, c_{j}, s\right)=\left(r c_{j}\right) s-$ $r\left(c_{j} s\right)=\left(c_{j} r\right) s-r\left(s c_{j}\right)=c_{j}(r s)-(r s) c_{j}=0$. Hence $c_{j} \in N_{m}(R)$.

Remark 29. Our proof of Theorem 6 (d)(e) follows closely the proof of Amitsur [2, Theorems 3 and 4] from the associative situation. We also remark that Amitsur's proof is much simpler in characteristic $p>0$ than the proofs given later by Jordan [15, Theorem 4.1.6] in the $\delta$-simple situation, although, as we show, Amitsur's original proof can be adapted to this situation.

\section{Non-Associative Weyl Algebras}

In this section, we show that there are lots of natural examples of non-associative differential polynomial rings. To this end, we introduce non-associative versions of the first Weyl algebra (see Definition 30) and we show that they are often simple regardless of the characteristic (see Theorem 32). Throughout this section, $T$ denotes a non-associative ring and $T[Y]$ denotes the polynomial ring over the indeterminate $Y$. In other words $T[Y]=T\left[Y ; \operatorname{id}_{R}, 0\right]$ as a generalized polynomial ring.

Definition 30. If $\delta: T[Y] \rightarrow T[Y]$ is a $T$-linear map such that $\delta(1)=0$, then the nonassociative differential polynomial $\operatorname{ring} T[Y]\left[X ; \mathrm{id}_{R}, \delta\right]$ is called a non-associative Weyl algebra.

Remark 31. A non-associative Weyl algebra is a generalization of the classical (associative) first Weyl algebra, hence the name. Recall that the first Weyl algebra, $A_{1}(\mathbb{C})=$ $\mathbb{C}\langle X, Y\rangle /(X Y-Y X-1)$ may be regarded as a differential polynomial ring $\mathbb{C}[Y]\left[X ; \mathrm{id}_{\mathbb{C}}, \delta\right]$, where $\delta: \mathbb{C}[Y] \rightarrow \mathbb{C}[Y]$ is the standard derivation on $\mathbb{C}[Y]$.

Theorem 32. If $T$ is simple and there for each positive $n \in \mathbb{N}$ is a non-zero $k_{n} \in Z(T)$ such that $\delta\left(Y^{n}\right)=k_{n} Y^{n-1}$, then the non-associative Weyl algebra $T[Y]\left[X ; \mathrm{id}_{R}, \delta\right]$ is simple. 
Proof. Put $R=T[Y]$ and $S=R\left[X ; \mathrm{id}_{R}, \delta\right]$. First we show that $R$ is $\delta$-simple. Let $I$ be a non-zero $\delta$-invariant ideal of $R$. Take a non-zero $a \in I$. Suppose that the degree of $a$ is $n$. From the definition of $\delta$ it follows that $\delta^{n}(a)$ is a non-zero element of $I$ of degree zero. This means that $I \cap T$ is non-zero. By simplicity of $T$ it follows that $I \cap T=T$. In particular $1 \in T=I \cap T \subseteq I$. Hence $I=R$.

It is clear that $\delta$ is a kernel derivation. Therefore, by Theorem 6 (b)(c) we are done if we can show that every non-zero monic $b \in R_{\delta}[X] \cap Z(S)$ is of degree zero. It is clear that $R_{\delta}=T$. Therefore $b \in T[X] \cap Z(S)$. Seeking a contradiction, suppose that the degree of $b$ is $n>0$. Put $b=X^{n}+c X^{n-1}+$ [lower terms]. From $b \in Z(S)$ it follows that $c \in Z(T)$. Take $r \in R$. Then $0=b r-r b=(\delta(r)+c r-r c) X^{n-1}+[$ lower terms $]=[c \in Z(T)]=\delta(r) X^{n-1}+[$ lower terms]. Thus $\delta(r)=0$, for all $r \in R$, which is a contradiction since e.g. $\delta(Y)=k_{1} \neq 0$.

Corollary 33. If $T$ is simple and $\delta$ is the classical derivative on $T[Y]$, then the non-associative Weyl algebra $T[Y]\left[X ; \mathrm{id}_{R}, \delta\right]$ is simple if and only if $\operatorname{char}(T)=0$.

Proof. The "if" statement follows immediately from Theorem 32 where $k_{n}=n$, for $n>0$.

Now we show the "only if" statement. Suppose that $\operatorname{char}(T)=p>0$. Then $Y^{p} \in$ $Z\left(T[Y]\left[X ; \mathrm{id}_{R}, \delta\right]\right)$. In particular, from Proposition 26. we get that $Z\left(T[Y]\left[X ; \mathrm{id}_{R}, \delta\right]\right)$ is not a field. By Theorem 6 (c) we get that $T[Y]\left[X ; \mathrm{id}_{R}, \delta\right]$ is not simple. As an alternative proof it is easy to see that the proper non-zero ideal in $T[Y]$ generated by $Y^{p}$ is $\delta$-invariant. Thus $T[Y]$ is not $\delta$-simple. By Theorem $6(\mathrm{c})$, we get that $T[Y]\left[X ; \mathrm{id}_{R}, \delta\right]$ is not simple.

\section{Kernel Derivations Defined by Automorphisms}

In this section, we show simplicity results for a differential polynomial ring version of the quantum plane (see Theorem 37) and for differential polynomial rings defined by actions on compact Hausdorff spaces (see Theorem 42). To this end, we introduce a class of $\sigma$-kernel derivations defined by ring morphisms (see Definition 34). Throughout this section, $R$ denotes a non-associative ring.

Proposition 34. If $\alpha: R \rightarrow R$ is a ring morphism, then the map $\delta_{\alpha}: R \rightarrow R$ defined by $\delta_{\alpha}(r)=\alpha(r)-r$, for $r \in R$, is a left and right $R_{\delta_{\alpha}}^{\mathrm{id}_{R}}$-linear $\alpha$-kernel derivation. Moreover, an ideal $I$ of $R$ is $\delta_{\alpha}$-simple if and only if it is $\alpha$-simple.

Proof. It follows immediately that $\delta_{\alpha}(1)=0$ and that $\delta_{\alpha}$ is additive. Now we will show that $\delta_{\alpha}$ in fact is $R_{\delta_{\alpha}}^{\mathrm{id}_{R}}$-linear both from the left and the right. In particular, $\delta_{\alpha}$ is an $\alpha$-kernel derivation. Take $r \in R$ and $s \in \operatorname{ker}\left(\delta_{\alpha}\right)$. Then $\delta_{\alpha}(r s)=\alpha(r s)-r s=\alpha(r) \alpha(s)-r s=$ $\alpha(r) s-r s=(\alpha(r)-r) s=\delta_{\alpha}(r) s$. In the same way we get that $\delta_{\alpha}(s r)=s \delta_{\alpha}(r)$. The last statement is clear since if $a \in I$, then $\delta_{\alpha}(a) \in I$ if and only if $\alpha(a)-a \in I$.

Remark 35. The $\alpha$-kernel derivation $\delta_{\alpha}$ from Proposition 34 is seldom a derivation. In fact, suppose that $\delta_{\alpha}$ is a derivation. Take $r, s \in R$. Then the relation $\delta_{\alpha}(r s)=\delta_{\alpha}(r) s+r \delta_{\alpha}(s)$ may be rewritten as $\delta_{\alpha}(r) \delta_{\alpha}(s)=0$. So in particular, we get that $\delta_{\alpha}(r)^{2}=0$. Hence, if $R$ is a reduced ring, i.e. a ring with no non-zero nilpotent elements, then $\delta_{\alpha}$ is a derivation if and only if $\alpha=\mathrm{id}_{R}$. Thus, $\delta_{\alpha}$ would have to be the zero map.

Let $T$ be a simple non-associative ring and suppose that $q \in Z(T) \backslash\{0\}$. Let $T[Y]$ denote the polynomial ring in the indeterminate $Y$ over $T$. Define a ring automorphism $\alpha_{q}: T[Y] \rightarrow T[Y]$ by the $T$-algebra extension of the relation $\alpha_{q}(Y)=q Y$. By Proposition 34, $\alpha_{q}$ in turn defines an $\alpha$-kernel derivation $\delta_{\alpha_{q}}: T[Y] \rightarrow T[Y]$. It is not hard to show, using Remark 35, that $\delta_{\alpha_{q}}$ is a classical derivation if and only if $q$ is nilpotent. 
Proposition 36. If $T$ is simple, then $T[Y]$ is $\delta_{q}$-simple if and only if $q$ is not a root of unity.

Proof. Put $R=T[Y]$. First we show the "only if" statement. Suppose that $q$ is a root of unity. Take a non-zero $n \in \mathbb{N}$ with $q^{n}=1$. Then the ideal of $R$ generated by $Y^{n}$ is $\alpha_{q^{-}}$simple. Thus, $R$ is not $\alpha_{q}$-simple. By Proposition 34 we get that $R$ is not $\delta_{\alpha_{q}}$-simple. Now we show the "if" statement. Suppose that $q$ is not a root of unity. Take a non-zero $\delta_{\alpha_{q}}$-invariant ideal $I$ of $R$. We wish to show that $I=R$. By Proposition $34 I$ is $\alpha_{q}$-invariant. Take a non-zero $a \in I$ of least degree $m$. Seeking a contradiction, suppose that $m>0$. Write $a=\sum_{i=0}^{m} a_{i} Y^{i}$, for some $a_{i} \in T$, for $i \in\{0, \ldots, n\}$. Then $\alpha_{q}(a)-k^{m} a$ is a non-zero element of $I$ of degree less than $m$. This contradicts the minimality of $m$. Thus $m=0$ and thus $a \in I \cap T$. Since $T$ is simple, we get that the ideal $J$ of $T$ generated by $a$ equals $T$. In particular, we get that $I \supseteq T \ni 1$. Thus $I=R$.

Theorem 37. If $T$ is simple and $\operatorname{char}(R)=0$, then the non-associative differential polynomial ring $D=T[Y]\left[X ; \mathrm{id}_{T[Y]}, \delta_{\sigma_{q}}\right]$ is simple if and only if $q$ is not a root of unity. In that case, $Z(D)=Z(T)$.

Proof. The "only if" statement follows from Theorem 6) (c) and Proposition 36. Now we show the "if" statement. Put $R=T[Y]$ and $\delta=\delta_{\alpha_{q}}$. Suppose that $q$ is not a root of unity. By Proposition [36, we get that $R$ is $\delta$-simple. By Theorem 6(c), we are done if we can show that $Z(S)$ is a field. To this end, we first note that, by Theorem 6 (b), there is a unique monic $b \in Z(D)$ of least degree $n$. Seeking a contradiction, suppose that $n>0$. Then $b=\sum_{i=0}^{n} b_{i} X^{i}$, for some $b_{i} \in R_{\delta}$. But since $q$ is not a root of unity, it follows that $R_{\delta}=T$. Thus $b \in T[X]$. From the fact that $b \in Z(D)$, we get that $b t=t b$, for $t \in T$, which in turn implies that $b_{i} \in Z(T)$, for $i \in\{0, \ldots, n\}$. By looking at the degree $n-1$ coefficient in the relations $b r=r b$, for $r \in R$, we get that $\alpha_{q}=\mathrm{id}_{R}$, which contradicts the fact that $q \neq 1$. Thus $n=0$ and it follows that $b=1$. By Theorem [6(b), we get that $Z(D)=Z(R)_{\delta}[1]=Z(T)$.

Remark 38. Given a field $\mathbb{F}$ and $q \in \mathbb{F} \backslash\{0\}$, we may define the so called quantum plane (see e.g. [16, Chapter IV]) as $\mathbb{F}_{q}[X, Y]=\mathbb{F}\langle X, Y\rangle /(Y X-q X Y)$. The quantum plane is an associative algebra and it can be realized as a classical Ore extension. Indeed, if we define $\sigma: \mathbb{F}[X] \rightarrow \mathbb{F}[X]$ by $\sigma(X)=q X$, then the quantum plane $\mathbb{F}_{q}[X, Y]$ is isomorphic to the Ore extension $\mathbb{F}[X][Y, \sigma, 0]$. While the quantum plane can be seen as a $q$-deformation, the non-associative Ore extension $D=T[Y]\left[X ; \mathrm{id}_{T[Y]}, \delta_{\sigma_{q}}\right]$ that we study in Theorem 37 can be seen as a non-associative deformation of the plane.

There are several ways to associate an (associative) algebra to a dynamical system $(G, X)$, where $G$ is a group acting on a topological space $X$. By associating a skew group algebra (see [21]) or a crossed product $C^{*}$-algebra (see [25]) to the dynamical system, it is possible to encode the dynamical system into the algebra in such a way that dynamical features (faithfulness, freeness, minimality etc) of the dynamical system correspond to algebraical properties of the algebra. We shall now show how to associate a non-associative differential polynomial ring to a dynamical system and exhibit a correspondence between minimality of the dynamical system and simplicity of the non-associative ring.

For the rest of this section, let $K$ denote any of the real algebras $\mathbb{R}$ (real numbers), $\mathbb{C}$ (complex numbers), $\mathbb{H}$ (Hamilton's quaternions), $\mathbb{O}$ (Graves' octonions), $\mathbb{S}$ (sedenions), etc. obtained by iterating the classical Cayley-Dickson doubling procedure of the real numbers (for more details concerning this construction, see e.g. [3]). It is well known that $K$ is then a reduced ring. Also, apart from the cases when $K$ equals $\mathbb{R}, \mathbb{C}$ or $\mathbb{H}, K$ is not associative. 
Furthermore, there is an $\mathbb{R}$-linear involution ${ }^{-}: K \rightarrow K$ and a norm $|\cdot|: K \rightarrow \mathbb{R}_{\geq 0}$ satisfying $k \bar{k}=|k|^{2}$, for $k \in K$. For the rest of this section, let $Y$ be a compact Hausdorff space and let $g: Y \rightarrow Y$ be a continuous map. A closed subspace $Z$ of $Y$ is called $g$-invariant if $g(Z) \subseteq Z$. The action of $g$ on $Y$ is called minimal if $\emptyset$ and $Y$ are the only $g$-invariant subspaces of $Y$. By abuse of notation, we let $C(Y)$ denote the ring of continuous functions $Y \rightarrow K$. Since $K$ is reduced, we get that $C(Y)$ is also reduced. The homeomorphism $g: Y \rightarrow Y$ defines a ring homomorphism $\sigma(g): C(Y) \rightarrow C(Y)$, where $\sigma(g)(f)=f \circ g$, for $f \in C(Y)$. By Proposition 34. $\sigma(g)$ in turn defines a $\sigma$-kernel derivation $\delta_{\sigma(g)}: C(Y) \rightarrow C(Y)$. Note that, by Remark 35, $\delta_{\sigma(g)}$ is a classical derivation if and only if $g=\mathrm{id}_{Y}$.

Proposition 39. If the action of $g$ on $Y$ is minimal, then the ring $C(Y)$ is $\delta_{\sigma(g)}$-simple.

Proof. Suppose that $Y$ is $g$-minimal. We show that $C(Y)$ is $\delta_{\sigma(g)}$-simple. Suppose that $I$ is a non-zero $\delta_{\sigma(g)}$-invariant ideal of $C(Y)$. For a subset $J$ of $I$ define $N_{J}=\cap_{f \in J} f^{-1}(0)$. Since $I$ is $\sigma(g)$-invariant it follows that $N_{I}$ is $g$-invariant.

It is clear that $N_{J}$ is closed. Since $I$ is non-zero it follows that $N_{I}$ is a proper subset of $Y$. By $g$-minimality of $Y$, we get that $N_{I}$ is empty. By compactness of $X$ we get that there is some finite subset $J$ of $I$ such that $N_{J}$ is empty. Define $h \in I$ by $h=\sum_{f \in J} f \bar{f}=\sum_{f \in J}|f|^{2}$. Since $N_{J}$ is empty we get that $h(x) \neq 0$ for all $x \in X$. Therefore $I$ contains the invertible element $h$ and hence $I=C(Y)$.

Theorem 40. The non-associative differential polynomial ring $D=C(Y)\left[X ; \operatorname{id}_{C(Y)}, \delta_{\sigma(g)}\right]$ is simple if the action of $g$ on $Y$ is minimal and the topology on $Y$ is non-discrete. In that case, $Z(D)=\mathbb{C}$, if $K=\mathbb{C}$, and $Z(D)=\mathbb{R}$, otherwise.

Proof. Put $R=C(Y)$. Suppose that the action of $g$ on $Y$ is minimal. By Proposition 39 we get that $R$ is $\delta_{\sigma(g)}$-simple. By Theorem 6 (c) we are done if we can show that $Z(D)$ is a field. To this end, we first note that, by Theorem 6(b), there is a unique monic $b \in Z(D)$ (up to addition of elements of $Z(R)_{\delta}$, which are of degree 0 ) of least degree $n$. Seeking a contradiction, suppose that $n>0$. Then $b=\sum_{i=0}^{n} b_{i} X^{i}$, for some $b_{i} \in R_{\delta_{\sigma(g)}}$. Take $i \in\{0, \ldots, n\}$ and $k_{i} \in b_{i}(Y)$. Since $b_{i} \in R_{\delta_{\sigma(g)}}$, we get that the set $b_{i}^{-1}\left(k_{i}\right)$ is non-empty and $g$-invariant. By $g$-minimality of $Y$, we get that $Y=b_{i}^{-1}\left(k_{i}\right)$, i.e. $b_{i}$ is the constant function $k_{i}$. Thus $b=\sum_{i=0}^{n} k_{i} X^{i}$. From the fact that $b \in Z(D)$, we get that $b k=k b$, for $k \in K$, which in turn implies that $b_{i} \in Z(K)$, for $i \in\{0, \ldots, n\}$. By looking at the degree $n-1$ coefficient in the relations $b r=r b$, for $r \in R$, we get that $\sigma(g)=\mathrm{id}_{R}$. Since the topology on $Y$ is non-discrete, this contradicts $g$-minimality of $Y$. Thus $n=0$ and it follows that $b=1$. Thus, by Theorem 6 (b), we get that $Z(D)=Z(R)_{\delta_{\sigma(g)}}[1]=Z(K)$. It is well known that $Z(K)=\mathbb{R}$ for all $K$ except $K=\mathbb{C}$.

Proposition 41. Suppose that $g: Y \rightarrow Y$ is a homeomorphism. The ring $C(Y)$ is $\delta_{\sigma(g)}$-simple if and only if the action of $g$ on $Y$ is minimal.

Proof. The "if" statement follows from Proposition 39

Now we show the "only if" statement. Suppose that $C(Y)$ is $\delta_{\sigma(g)}$-simple. We show that $Y$ is $g$-minimal. Suppose that $Z$ is a closed $g$-invariant subset of $Y$ with $Z \subsetneq Y$. We wish to show that $Z=\emptyset$. To this end, let $I_{Z}$ denote the set of continuous functions $X \rightarrow \mathbb{C}$ that vanish outside $Z$. It is clear that $I_{Z}$ is an ideal of $C(Y)$. It is also clear that $I_{Z} \subsetneq C(Y)$ since all non-zero constant maps belong to $C(Y) \backslash I_{Z}$. Now we show that $I_{Z}$ is $\delta_{\sigma(g)}$-invariant. Take $f \in I_{Z}$ and $x \in Y \backslash Z$. Then $\delta_{\sigma(g)}(f)(x)=\sigma(g)(f)(x)-f(x)=\left[f \in I_{Y} \Rightarrow f(x)=\right.$ $0]=\sigma(g)(f)(x)=f(g(x))=0$. The last equality follows since $g(x) \in Y \backslash Z$. Now we prove 
this. Seeking a contradiction, suppose that $g(x) \in Z$. Then, by the $g$-invariance of $Z$, we get $g^{-1}(Z)=Z$, and $x=g^{-1}(g(x)) \in Z$, which is a contradiction. By $\delta_{\sigma(g)}$-simplicity of $C(Y)$ this implies that $I_{Z}=\{0\}$. Since $Y$ is compact, it is completely regular. Therefore, we get that $Z=\emptyset$.

Theorem 42. Suppose that $g: Y \rightarrow Y$ is a homeomorphism. The non-associative differential polynomial ring $D=C(Y)\left[X ; \operatorname{id}_{C(Y)}, \delta_{\sigma(g)}\right]$ is simple if and only if the action of $g$ on $Y$ is minimal and the topology on $Y$ is non-discrete. In that case, $Z(D)=\mathbb{C}$, if $K=\mathbb{C}$, and $Z(D)=\mathbb{R}$, otherwise.

Proof. Put $R=C(Y)$. The "if" statement follows from Theorem 40 ,

Now we show the "only if" statement. Suppose that $S$ is simple. By Theorem 6] and Proposition 41. it follows that the action of $g$ on $Y$ is minimal. Seeking a contradiction, suppose that the topology on $Y$ is discrete. Since the topology is Hausdorff it follows that $Y$ is a one-element set. Thus $S$ equals the polynomial ring $K[X]$ which is not simple.

\section{Associative Coefficients}

In this section, we show that if the ring of coefficients is associative, then we can often obtain simplicity of the differential polynomial ring just from the assumption that the map $\delta$ is not a derivation.

Theorem 43. Suppose that $D=R\left[X ; \mathrm{id}_{R}, \delta\right]$ is a non-associative differential polynomial ring such that $R$ is associative and all positive integers are regular in $R$. If $R$ is $\delta$-simple but $\delta$ is not a derivation, then $D$ is simple.

Proof. Let $I$ be a non-zero ideal of $D$. We wish to show that $I=D$. Pick a non-zero element $b \in I$ of least degree $n$. Let $b=\sum_{i=0}^{n} c_{i} X^{i}$, for some $c_{0}, \ldots, c_{n} \in R$. By mimicking the proof of Theorem 6(a), we can conclude that we may choose $c_{n}=1$. Seeking a contradiction, suppose that $n>0$. We claim that $(b, d, e)=0$, for all $d, e \in R$. If we assume that the claim holds, then by extracting the terms of degree $n-1$ from the relation $(b, d, e)=0$ we get that $n d \delta(e)+n \delta(d) e+\left(c_{n-1} d\right) e-n \delta(d e)-c_{n-1}(d e)=0$. But since $R$ is associative and $n$ is regular, this implies that $d \delta(e)+\delta(d) e=\delta(d e)$ which contradicts the fact that $\delta$ is not a derivation. Thus $n=0$ and hence $1=b \in I$ which in turn implies that $I=D$. Now we show the claim. The degree $n$ part of $(b, d, e)$ equals $\left(c_{n} d\right) e-c_{n}(d e)=(1 \cdot d) e-1 \cdot(d e)=0$. Thus, since $(b, d, e) \in I$, we get that $(b, d, e)=0$, from the minimality of $n$.

Remark 44. In the cases when $T$ is associative, i.e. in the cases when $K=\mathbb{R}, K=\mathbb{C}$ or $K=\mathbb{H}$, then Theorem 43 can be used to simplify the proofs of Theorem 37 and Theorem 42 .

\section{REFERENCES}

[1] S. A. Amitsur, Finite differential polynomials, Riveon Lematematika 4, 1-8 (1950).

[2] S. A. Amitsur, Derivations in simple rings, Proc. London Math. Soc. (3) 7, 87-112 (1957).

[3] J. C. Baez, The octonions, Bull. Amer. Math. Soc. (N.S.) 39(2), 145-205 (2002).

[4] G. M. Bergman, A ring primitive on the right but not on the left, Proc. Amer. Math. Soc. 15, 473-475 (1964).

[5] G. M. Bergman, The diamond lemma for ring theory, Adv. in Math. 29(2), 178-218 (1978).

[6] P. M. Cohn, Quadratic extensions of skew fields, Proc. London Math. Soc. (3) 11, 531-556 (1961).

[7] P. M. Cohn, Skew field constructions, London Mathematical Society Lecture Notes Series, No. 27, Cambridge University Press, Cambridge-New York-Melbourne (1977).

[8] J. Cozzens and C. Faith, Simple Noetherian rings, Cambridge Tracts in Mathematics, No. 69, Cambridge University Press, Cambridge-New York-Melbourne (1975). 
[9] N. J. Fine, Binomial coefficients modulo a prime, Amer. Math. Monthly 54, 589-592 (1947).

[10] K. R. Goodearl, Centralizers in differential, pseudo-differential, and fractional differential operator rings, Rocky Mountain J. Math. 13(4), 573-618 (1983).

[11] K. R. Goodearl and R. B. Warfield, Primitivity in differential operator rings, Math. Z. 180(4), 503-523 (1982).

[12] L. Hellström and S. D. Silvestrov, Commuting elements in q-deformed Heisenberg algebras, World Scientific Publishing Co., River Edge, NJ (2000).

[13] N. Jacobson, Pseudo-linear transformations, Ann. of Math. (2) 38(2), 484-507 (1937).

[14] N. Jacobson, Finite-dimensional division algebras over fields, Springer-Verlag, Berlin (1996).

[15] D. A. Jordan, Ore extensions and Jacobson rings, Ph.D. thesis, University of Leeds (1975).

[16] C. Kassel, Quantum groups, Graduate Texts in Mathematics, No. 155, Springer-Verlag, New York (1995).

[17] D. R. Malm, Simplicity of partial and Schmidt differential operator rings, Pacific J. Math. 132(1), 85-112 (1988).

[18] J. C. McConnell and J. C. Robson, Noncommutative Noetherian Rings, Graduate Studies in Mathematics, No. 30, American Mathematical Society, Providence, RI (2001).

[19] P. Nystedt, A combinatorial proof of associativity of Ore extensions, Discrete Math. 313(23), 2748-2750 (2013).

[20] P. Nystedt, J. Öinert and J. Richter, Iterated Non-associative Ore Extensions, preprint in preparation.

[21] J. Öinert, Simplicity of skew group rings of abelian groups, Comm. Algebra 42(2), 831-841 (2014).

[22] J. Öinert, J. Richter and S. D. Silvestrov, Maximal commutative subrings and simplicity of Ore extensions, J. Algebra Appl., 12(4), 1250192, 16 pp. (2013).

[23] O. Ore, Theory of non-commutative polynomials, Ann. of Math. (2) 34(3), 480-508 (1933).

[24] E. C. Posner, Differentiably simple rings, Proc. Amer. Math. Soc. 11, 337-343 (1960).

[25] S. C. Power, Simplicity of $C^{*}$-algebras of minimal dynamical systems, J. London Math. Soc. (2) 18(3), 534-538 (1978).

[26] J. Richter, A Note on "A combinatorial proof of associativity of Ore extensions", Discrete Math. 315, 156-157 (2014).

[27] L. H. Rowen, Ring Theory, Vol. I, Pure and Applied Mathematics, No. 127, Academic Press, Boston, MA (1988).

[28] M. G. Voskoglou, Simple skew polynomial rings, Publ. Inst. Math. (Beograd) (N.S.), 37(51), 37-41 (1985). 